M 1 Raabe Einfübrung in die Bücherkunde

M 2 Meisen Altdeutsche Grammatik 1: Lautlebre

M 3 Meisen Altdeutsche Grammatik II: Formenlebre

M 4 Grimm Bertolt Brecht

M 5 Moser Annalen der deutschen Sprache

M 6 Schlawe Literarische Zeitschriften 1885-1910

M 7 Weber/Hoffmann Nibelungenlied

M 8 Meyer Eduard Mörike

M 9 Rosenfeld Legende

M 10 Singer Der galante Roman

M 12 Nagel Meistersang

M 13 Bangen Die schriftliche Form germanist. Arbeiten

M 14 Eis Mittelalterliche Facbliteratur

M 15 Weber/Hoffmann Gottfried von Straßburg

M 16 Lüthi Märchen

M 17 Wapnewski Hartmann von Aue

M 18 Meetz Friedrich Hebbel

M 19 Schröder Spielmannsepik

M 20 Ryan Friedrich Hölderlin

M 22 Danzel Zur Literatur und Philosophie der Goethezeit

M 24 Schlawe Literarische Zeitschriften 1910-1933

M 25 Anger Literarisches Rokoko

M 26 Wodtke Gottfried Benn

M 27 von Wiese Novelle

M 28 Frenzel Stoff-, Motiv- und Symbolforschung

M 29 Rotermund Christian Hofmann von Hofmannswaldau

M 30 Galley Heinrich Heine

M 31 Müller Franz Grillparzer

M 32 Wisniewski Kudrun

M 33 Soeteman Deutsche geistliche Dichtung des 11.u. 12. Jh.s

M 34 Taylor Melodien der weltlichen Lieder des Mittelalters I: Darstellung

M 35 Taylor Melodien der weltlichen Lieder des Mittelalters II: Materialien

M 36 Bumke Woltram von Eschenbach

M 37 Engel Handlung, Gespräch u. Erzäblung. Faksimiledruck

M 38 Brogsitter Artuseptik

M 39 Blankenburg Versuch über den Roman. Faksimiledruck 


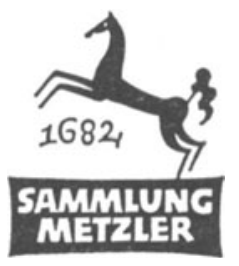

REALIEN ZUR LITERATUR

ABT. D:

LITERATURGESCHICHTE 
SVEN-AAGE JØRGENSEN

\section{Johann Georg Hamann}

MCMLXXVI

J. B.METZLERSCHE VERLAGSBUCHHANDLUNG

STUTTGART 
ISBN 978-3-476-10143-3

ISBN 978-3-476-03853-1 (eBook)

DOI 10.1007/978-3-476-03853-1

M 143

(C) Springer-Verlag GmbH Deutschland 1976

Ursprünglich erschienen bei J. B. Metzlersche Verlagsbuchhandlung und Carl Ernst Poeschel Verlag GmbH in Stuttgart 1976 


\section{INHALT}

I. Materialien (Werk- und Briefausgaben, Bio- und Bibliographien, Literaturübersicht . . . . . . . . . 1

II. Leben und Werk . . . . . . . . . . . . . . . . . 15

1. Jugend in Königsberg . . . . . . . . . . . . 15

2. Hofmeister und Kaufmann . . . . . . . . . . 23

3. Der Königsberger Sokrates . . . . . . . . . . . 38

a) Der neue Stil . . . . . . . . . . . . . 38

b) "Sokratische Denkwürdigkeiten« . . . . . . . . 43

c) »Wolken« und Geniebegriff . . . . . . . . . . 45

4. Kreuzzüge des Philologen . . . . . . . . . . . 50

5. Der Zöllner und die Magd . . . . . . . . . . . . . 61

6. Sprache . . . . . . . . . . . . . 66

7. Politik ................ 76

8. Mysterien . . . . . . . . . . . . . 80

9. Prediger in der Wüsten . . . . . . . . . . . . 87

10. Reise nach Westfalen, Tod . . . . . . . . . 93

III. Nachwirkung und Stand der Forschung . . . . . . 98

Register . . . . . . . . . . . . . . . . . . . 104 


\section{VERZEICHNIS DER \\ ABGEKURZT ANGEFUHRTEN LITERATUR}

Accolti: Accolti Gil Vitale Nicola: La giovinezza di Hamann. Varese 1957; ${ }^{2} 1960$.

Baudler: Baudler, G:»Im Worte sehen.« Das Sprachdenken J. G. Hamanns. Bonn 1970.

Blanke: Blanke, Fritz: Hamann-Studien. Zürich 1956.

Blum: Blum, Jean: La vie et l'œuvre de J. G. Hamann, le »Mage du Nord«, 1730 - 1788. Paris 1912.

Gajek: Gajek, Bernhard: Sprache beim jungen Hamann. Diss. München 1959. Bern ${ }^{2} 1967$.

Gründer: Gründer, Karlfried: Figur und Geschichte. Johann Georg Hamanns "Biblische Betrachtungen* als Ansatz einer Geschichtsphilosophie. In: Symposion. Philosophische Schriftenreihe. Nr. 3. Freiberg/München 1958.

Hamannausg.: Nadler, Josef: Die Hamannausgabe. Vermächtnis, Bemühungen, Vollzug. Halle. 1930.

Hoffmann: Hoffmann, V.: J. G. Hamanns Philologie. Hamanns Philologie zwischen enzyklopädischer Mikrologie und Hermeneutik. Stuttgart 1972.

Jansen Schoonhoven: Jansen Schoonhoven, Evert: Natuur en Genade bij J. G. Hamann. Nijkerk 1945.

Jørgensen: Hamann, Johann Georg: Fünf Hirtenbriefe das Schuldrama betreffend. Einführung und Kommentar von Sven-Aage Jørgensen. København. 1962. (=Historisk-filosofiske Meddelelser udgivet af Det Kongelige Danske Videnskabernes Selskab Bind 39, nr. 5).

Knoll: Knoll, Renate: Johann Georg Hamann und Friedrich Heinrich Jacobi. Heidelberg 1963.

Koepp: Koepp, Wilhelm: Der Magier unter Masken. Versuch eines neuen Hamannbildes. Göttingen 1965.

Lumpp: Lumpp, H. M.: Philologia crucis. Zu J. G. Hamanns Auffassung von der Dichtkunst. Mit einem Kommentar zur Aestetica in nuce. Tübingen 1970.

Manegold: Manegold, Ingemarie: Johann Georg Hamanns Schrift "Konxompax«. Fragmente einer apokryphischen Sibylle über apokalyptische Mysterien. Text, Entstehung und Bedeutung. Heidelberg 1963.

Metzke: Metzke, Erwin: J. G. Hamanns Stellung in der Philosophie des 18. Jahrhunderts. Halle 1934. Reprografischer Nachdruck. Darmstadt 1967.

Minor: Minor, Jacob: Hamann in seiner Bedeutung für die Sturm- und Drangperiode. Frankfurt/M. 1881.

Ruprecht: Ruprecht, Erich: Der Aufbruch der romantischen Bewegung. München 1948.

Salmony: Salmony, Hansjörg Alfred: Johann Georg Hamanns metakritische Philosophie. Zollikon 1958.

Schack: Schack, Tage: Johann Georg Hamann. Kopenhagen o. J. [1948]. Schneider: Sturm und Drang. Kritische Schriften. Für Anmerkungen und 
Register sind verantwortlich: Lambert Schneider und Waltraut Schleuning. Heidelberg o. J.

Schreiner: Schreiner, Helmuth: Die Menschwerdung Gottes in der Theologie Johann Georg Hamanns. Tübingen 1946. 2. erw. Auflage Tübingen 1950.

Seils: Seils, Martin: Theologische Aspekte zur gegenwärtigen Hamanndeutung. Berlin/Göttingen 1957.

Sievers: Sievers, Harry: Johann Georg Hamanns Bekehrung. Ein Versuch, sie zu verstehen. Zürich 1969.

Simon: J. G. Hamann: Schriften zur Sprache. Einleitung von Josef Simon. Frankfurt/M. 1967.

Strässle: Strässle, U.: Geschichte, geschichtliches Verstehen und Geschichtsschreibung im Verständnis J. G. Hamanns. Eine entwicklungsgeschichtliche Untersuchung d. Werke zwischen 1756 und 1772. Bern 1970.

Unger: Unger, Rudolf: Hamann und die Aufklärung, Bd. 1-2, Jena 1911. Wild: Wild, Reiner: >Metacriticus bonae spei<. Johann Georg Hamanns »Fliegender Brief«. Einführung, Text und Kommentar. Frankfurt/M. 1975.

\section{ABKURZUNGEN}

G Gildemeister, C. H., Johann Georg Hamann's, des Magus im Norden, Leben und Schriften, 6 Bde, Gotha 1857-73.

$\mathrm{HH}$ Johann Georg Hamann's Hauptschriften erklärt, hrsg. v. Fritz Blanke und Lothar Schreiner, Gütersloh $1956 \mathrm{f}$.

$\mathrm{Na}$ Nadler, Josef, Johann Georg Hamann. Der Zeuge des Corpus mysticum, Salzburg 1949.

R Hamann's Schriften, hrsg. v. Friedrich Roth, Bd. 1-7, Berlin 1821-1825; Bd. 8, 1-2, hrsg. v. G. A. Wiener, Berlin 1842-1843.

$\mathrm{ZH}$ Hamann, Johann Georg, Briefwechsel, bisher erschienen Bd. 1-3, hrsg. v. Walther Ziesemer und Arthur Henkel, Wiesbaden 19551957; Bd. 4-6, hrsg. v. Arthur Henkel, Wiesbaden 1959, Frankfurt 1975. 